\title{
Application of Light Scattering from a Dielectric Cylinder Based upon Mie and Rayleigh-Gans-Born Theories to Polymer Systems. III. An Application to Polymer Composites
}

\author{
Yukikazu Uemura, ${ }^{*}$ Takeji Hashimoto, and Hiromichi KAWAI \\ Department of Polymer Chemistry, Faculty of Engineering, \\ Kyoto University, Kyoto 606, Japan.
}

(Received November 1, 1978)

\begin{abstract}
Mie and Rayleigh-Gans-Born scattering theories from a dielectric and coaxial cylinder were applied to polymer-glass fiber composite systems. The size and refractive indices of the polymer-coated glass fiber were estimated by laser light scattering experiments. These light scattering methods were also applied to a glass-fiber rubber composite to analyze the debonding of a fiber from the polymer matrix when the composite was subjected to applied stress.
\end{abstract}

KEY WORDS Mie Scattering / Rayleigh-Gans-Born Scattering / Coated Glass Fiber / Polymer Composite / Coaxial Cylinder / Interfacial Debonding /

The technique of reinforcing polymers by fibers, especially glass fibers, has recently achieved remarkable progress. ${ }^{1}$ It is both very important and interesting from academic and practical points of view, to analyze the structure of the boundary between the fiber and polymer matrix. The adhesion or debonding of a polymer to the fiber at the boundary is closely related to the mechanical strength and failure of a composite.

Our purpose in this work is to study the boundary structure of the composite by means of light scattering technique. In Part $I,{ }^{2}$ we studied the application of Mie scattering, which is rigorous theory of electromagnetic scattering from scattering objects with large refractive-index differences to their surrounding medium, we evaluated the size and the refractive index of the glass fiber having a radius much larger than the wavelength of the incident beam. The analyses are based upon the Mie scattering and Rayleigh-Gans-Born (R.G.B.) scattering theories from a dielectric and circular cylinder of infinite length.

These analyses have been extended to the analyses based upon the dielectric, coaxial and circular cylinders in Part $\mathrm{II}^{3}$ so as to evaluate the size and refractive index of the sheath as well as those of the

* Present Address: Sumitomo Chemical Co. Ltd., 1-98, 3-chome Naka, Kasugade, Konohana-ku, Osaka 554, Japan. core. The scattering from such a system has many practical applications, e.g., the scattering from a glass fiber coated with silane coupling agents for which a coated sheath is introduced to improve the compatibility of the fiber with the polymer matrix, the scattering from crazes or cracks produced in the periphery of the fiber in the fiber-reinforced plastics (FRP) subjected to stress, the scattering from coated optical fibers, and etc. In Part II we are concerned, more or less, with evaluating parameters by numerically analyzing the effects of various parameters on scattering intensity distributions in a forward direction.

In this article we study the practical application of Mie scattering from the coaxial cylinder to a polymer-coated glass fiber and to a system in which the glass fiber is embedded in a crosslinked rubber (FRR). In the former system, the fiber was immersed in silicone oils having various refractive indices to facilitate the evaluation of the sheath parameters. ${ }^{2}$ In the latter system, scattering was detected as a function of the applied strain imposed perpendicularly to the fiber. These results are discussed in connection with the debonding of the polymer matrix from the fiber. The scattering from these systems is described in terms of a simplified model of a coaxial cylinder embedded in a rubber matrix, (the sheath corresponds to the debonded void- or the density lowered-phase). 
The effects of size distribution and finite length of the cylinder are also discussed briefly.

\section{EXPERIMENTAL}

The E-glass long monofilaments used in this study have a refractive index of 1.548 and radius of 2.5 or $5.0 \mu \mathrm{m}$. The parameters are determined by the method described in a previous article. ${ }^{2}$

A polymer-coated glass fiber was prepared by dipping the fiber in a $10-\mathrm{wt} \%$ toluene solution of polybutadiene. Uniformity of the coated layer of 1.0-3.0- $\mu \mathrm{m}$ thickness was checked by optical microscopy. The coated glass fiber was then used for light scattering experiments under a dry state condition or in immerson liquids of various refractive indices (silicone oils being used as an immersion liquid). The irradiated part of the fiber underwent no change throughout the experiment.

Scattering experiments were also conducted for fibers embedded in the rubber (crosslinked polybutadiene) matrix. Seven grams of polybutadiene (with high cis-content, BR-01, Japan Synthetic Rubber Co.) were dissolved in 45 grams of toluene with 0.14 grams of dicumeneperoxide to serve as a crosslinking agent. The solution was then cast on a glass plate on which a long glass fiber was fastened. The film prepared after drying was pressed under a laboratory hot press at $150^{\circ} \mathrm{C}$ for $30 \mathrm{~min}$ for crosslinking. The transparent film specimens of 2-mm wide and 50-mm long were then cut in such a way that the glass fiber was in the center and normal to the length of the film specimens. Scattering measurements were conducted also for specimens subjected to tensile elongation. The degree of elongation was measured by reading the distances between the marks written on the original sample.

A He-Ne gas laser (15-mW power with a single mode) was used as the plane polarized incident beam. The angular dependence of the scattered intensity was measured, by scanning a photomultiplier in a horizontal plane under the $V_{\mathrm{v}}$ polarization condition in which the polarization directions of the incident beam, analyzer and the axis of the glass fiber are set vertically. The measurements on the FRR films were conducted under the same conditions above and also by stretching the films in a horizontal direction. The photographic light scattering patterns were taken to make a quick investigation of all the scattering intensity distributions of the system.

\section{THEORETICAL BACKGROUND}

The intensity of Mie scattering from a coaxial cylinder with inner radius $R_{1}$, refractive index $m_{1}$ and outer radius $R_{2}$ and refractive index $m_{2}$ in the homogeneous medium $m_{3}$ in a direction perpendicular to the cylinder under the $V_{\mathrm{v}}$ condition for normal incidence, is given by ${ }^{4}$

$$
I_{V_{\mathrm{v}}}(\theta) / I_{0}=\frac{\lambda_{0}}{\pi^{2} \rho_{m_{3}}}\left|b_{0}+2 \sum_{n=1}^{\infty} b_{n} \cos (n \theta)\right|^{2} \mid
$$

where $\theta$ is the scattering angle, $I_{0}$ the intensity of the incident beam, $\lambda_{0}$ the wavelength of the incident beam in vacuo and $\rho$, the distance between the point of observation and the scattering material. The cylinder was assumed to be infinitely long and optically isotropic. The coefficients $b_{n}$ is given by

$$
b_{n}=\frac{\left|\begin{array}{llll}
J_{n}\left(m_{3} \alpha_{2}\right) & H_{n}\left(m_{3} \alpha_{2}\right) & J_{n}\left(m_{2} \alpha_{2}\right) & 0 \\
m_{3} J_{n}^{\prime}\left(m_{3} \alpha_{2}\right) & m_{2} H_{n}^{\prime}\left(m_{2} \alpha_{2}\right) & m_{2} J_{n}^{\prime}\left(m_{2} \alpha_{2}\right) & 0 \\
0 & H_{n}\left(m_{2} \alpha_{1}\right) & J_{n}\left(m_{2} \alpha_{1}\right) & J_{n}\left(m_{1} \alpha_{1}\right) \\
0 & m_{2} H_{n}^{\prime}\left(m_{2} \alpha_{1}\right) & m_{2} J_{n}^{\prime}\left(m_{2} \alpha_{1}\right) & m_{1} J_{n}^{\prime}\left(m_{1} \alpha_{1}\right)
\end{array}\right|}{\left|\begin{array}{llll}
H_{n}\left(m_{3} \alpha_{2}\right) & H_{n}\left(m_{2} \alpha_{2}\right) & J_{n}\left(m_{2} \alpha_{2}\right) & 0 \\
m_{3} H_{n}^{\prime}\left(m_{3} \alpha_{2}\right) & m_{2} H_{n}^{\prime}\left(m_{2} \alpha_{2}\right) & m_{2} J_{n}^{\prime}\left(m_{2} \alpha_{2}\right) & 0 \\
0 & H_{n}\left(m_{2} \alpha_{1}\right) & J_{n}\left(m_{2} \alpha_{1}\right) & J_{n}\left(m_{1} \alpha_{1}\right) \\
0 & m_{2} H_{n}^{\prime}\left(m_{2} \alpha_{1}\right) & m_{2} J_{n}^{\prime}\left(m_{2} \alpha_{1}\right) & m_{1} J_{n}^{\prime}\left(m_{1} \alpha_{1}\right)
\end{array}\right|}
$$

where

$$
\alpha_{1}=2 \pi R_{1} / \lambda_{0}, \alpha_{2}=2 \pi R_{2} / \lambda_{0}
$$

The functions $J_{n}$ and $H_{n}$ are the first kind Bessel and the second kind Hankel functions of the order $n$, and $J_{n}{ }^{\prime}$ and $H_{n}{ }^{\prime}$ are the respective first-order derivatives. The scattering efficiency $Q_{V_{\mathrm{v}}}$ difined as the ratio of the scattering cross section to the geometrical cross 
section of the scattering object, is also estimated as

$$
Q_{V_{\mathrm{v}}}=\frac{\lambda_{0}}{\pi R_{2}}\left(\left|b_{0}\right|^{2}+2 \sum_{n=1}^{\infty}\left|b_{n}\right|^{2}\right)
$$

The effect of a finite length of the fiber on the scattered intensity distribution occurs in the change of the meridional intensity distribution (i.e., the intensity distribution parallel to the fiber axis) without changing the equatorial intensity distribution, ${ }^{5}$ i.e., the intensity distribution $I_{V_{\mathrm{v}}}(\theta)_{\mathrm{f}}$ for the fiber with finite length is given by

$$
I_{V_{\mathrm{v}}}(\theta)_{\mathrm{f}} \sim j_{0}^{2}(k L \varphi / 2) I_{V_{\mathrm{v}}}(\theta)
$$

where $j_{0}$ is the zeroth order spherical Bessel function of the first kind, $L$, the length of the fiber and $\varphi_{1}$ the scattering angle in the meridional direction, and $k=2 \pi / \lambda$ ( $\lambda$ being the wavelength in the surrounding medium of the fiber). $I_{V_{\mathrm{v}}}(\theta)$ is given by eq 1 . Thus, the angle which gives rise to the first order scattering maximum along the meridian, is related to $L$ by

$$
L(\mu \mathrm{m})=0.9064 / \varphi(\mathrm{rad})
$$

The effect of the size distribution in the radius $R_{2}$ of the coaxial circular cylinder on the intensity distribution was qualitatively examined by assuming the zeroth order logarithmic distribution (ZOLD) ${ }^{4}$ function $P\left(R_{2}\right)$

$$
\begin{aligned}
P\left(R_{2}\right)= & \exp \left(-\frac{\left(\ln R_{2}-\ln R_{\mathrm{M}}\right)^{2}}{2 \Delta^{2}}\right) / \\
& \sqrt{2 \pi} \Delta R_{\mathrm{M}} \exp \left(\frac{\Delta^{2}}{2}\right)
\end{aligned}
$$

where $R_{\mathrm{M}}$ is the modal value of $R_{2}$, and $\Delta$ is the parameter related to the width and skewness of the distribution function. The distribution was used to analyze the void region developed at the boundary between the fiber and the rubber matrix in the stretched FRR films.

If the scattering from the coaxial cylinder is capable of being described in terms of the Rayleigh-Gans-Born (R.G.B.) or RayleighGans-Debye theory, the $V_{\mathrm{v}}$ scattering intensity may be given by ${ }^{3}$

$$
I_{V_{\mathrm{v}}}=\text { (const) }\left(\left(m_{1}-m_{2}\right) \frac{J_{1}\left(U_{1}\right)}{U_{1}}\right.
$$

$$
\begin{gathered}
\left.+\left(m_{2}-m_{3}\right)\left(\frac{R_{2}}{R_{1}}\right)^{2} \frac{J_{1}\left(U_{2}\right)}{U_{2}}\right)^{2} \\
U_{1}=(4 \pi / \lambda) R_{1} \sin (\theta / 2), U_{2}=(4 \pi / \lambda) R_{2} \sin (\theta / 2)
\end{gathered}
$$

The scattering maxima and the beating $\operatorname{maxima}^{3}$ are shown to occur, respectively, at the scattering angles $\theta_{\mathrm{m}}$ and $\theta_{\mathrm{mb}}$, satisfying the following conditions,

$$
\begin{aligned}
m\left(R_{1}+R_{2}\right) \sin \left(\theta_{m} / 2\right)= & \left(\lambda_{0} / 2 \pi\right)((\pi / 2) n+\beta) \\
& (n=1,3,5,7, \cdots) \\
m\left(R_{2}-R_{1}\right) \sin \left(\theta_{m b} / 2\right)= & \left(\lambda_{0} / 4\right) n \\
& (n=1,3,5,7, \cdots)
\end{aligned}
$$

where $\beta=2.35619$ and $m$ is the average refractive index of the system.

The eq 10 and 11 are derived under conditions such that

$$
m_{1} \simeq m_{3}, R_{2} \simeq R_{1}
$$

These relations may be also applied for estimating $R_{1}$ and $R_{2}$.

\section{RESULTS}

\section{Polymer-Coated Glass Fiber}

Figure 1 represents typical optical micrographs of the E-glass fiber of 5.0- $\mu \mathrm{m}$ diameter (a) and the fiber coated by polybutadiene (b). It is shown from the micrograph that the coated sheath has a uniform thickness. The coated fiber can thus be treated by the coaxial cylinder model. Figure 2 shows typical $V_{\mathrm{v}}$ light scattering patterns from (a) a glass fiber and from (b) a coated glass fiber. The scattering maxima are capable of being described in terms of the Mie theory to be discussed later. The maxima of the coated fiber occur at scattering angles smaller than those of the simple fiber. The coaxial fiber exhibits fine variations of scattered intensity with scattering maxima and minima along the meridional direction. These variations may reflect non-uniformity of the sheath thickness in a microscopic scale as will be discussed later.

Figure 3 shows a photometrically measured intensity distribution of a coated fiber having an inner radius $R_{1}=5 \mu \mathrm{m}$ and a refractive index of $m_{1}=1.548$ (open circles) in the air. The figure also shows the 


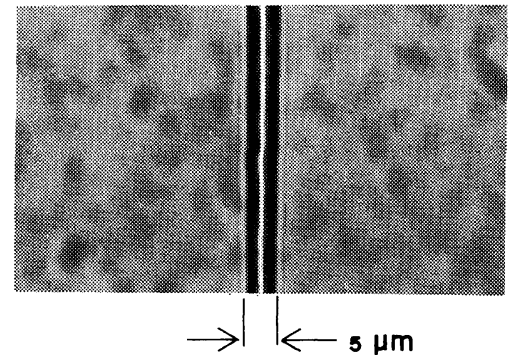

(a)

G.F.

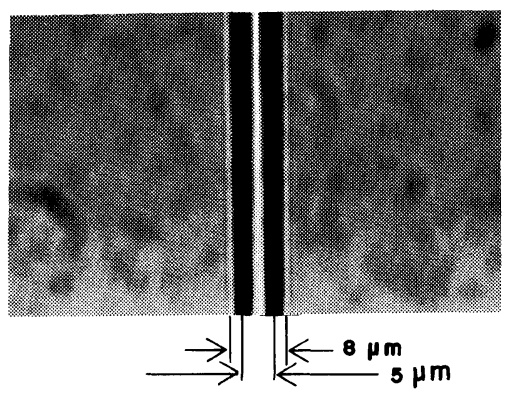

(b)

G.F. + P. B.

Figure 1. Typical optical micrographs of (a) the E-glass fiber and (b) the polybutadiene-coated glass fiber.

calculated Mie scattering from a simple glass fiber of $R_{1}=5 \mu \mathrm{m}$, and $m_{1}=1.548$ (broken line) and from a coaxial cylinder of the same values of $R_{1}$ and $m_{1}$ and the outer radius $R_{2}=5.5 \mu \mathrm{m}$ and refractive index $m_{2}=1.522$ (solid line). Also is shown the calculated R.G.B. scattering (dash-dot line) for the same set of the parameters. All the calculated curves are obtained for the scattering object in the air phase and this will be discussed later.

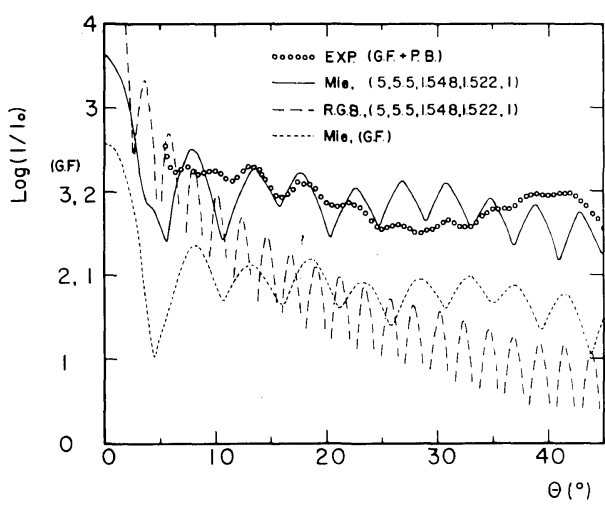

Figure 3. Photometrically measured scattered intensity distribution of the polybutadiene-coated glass fiber (open circles). The radius $R_{1}$ and refractive index $m_{1}$ of the fiber are $5.0 \mu \mathrm{m}$ and 1.548 , respectively. The solid and broken lines are the calculated Mie scattering intensity distributions from the coated glass fiber and from the glass fiber, respectively. The dash-dot line corresponds to the calculated R.G.B. scattering. The numbers in parentheses refer to the values of $\left(R_{1}, R_{2}, m_{1}, m_{2}, m_{3}\right)$ where $R_{2}$ is the radius of the outer cylinder, $m_{2}$ and $m_{3}$ are the refractive indices of the sheath and the surrounding medium, respectively.

Figure 4 shows the measured intensity distribution (open circle) of a coated fiber immersed in a silicone oil of a refractive index of $m_{3}=1.54$ and also the calculated Mie (solid line) and R.G.B. (dash-dot line) curves for the coaxial cylinder immersed in a medium of refractive index $m_{3}=1.54$. The calculated curves were obtained for the same set of parameters ( $R_{1}=5 \mu \mathrm{m}, R_{2}=5.5 \mu \mathrm{m}, m_{1}=1.548$ and $\left.m_{2}=1.522\right)$ as those shown in Figure 3.

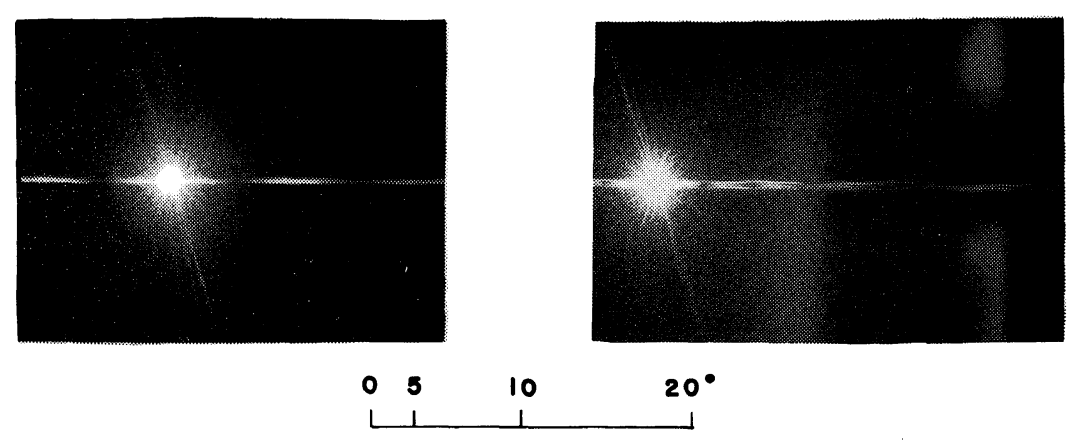

(a) G.F.

(b) G. F. + P.B.

Figure 2. Typical photographic light scattering patterns for (a) the glass fiber and (b) the polybutadienecoated glass fiber. 
Light Scattering from a Debonded Phase of the FRR The debonding of the glass fiber from the polymer

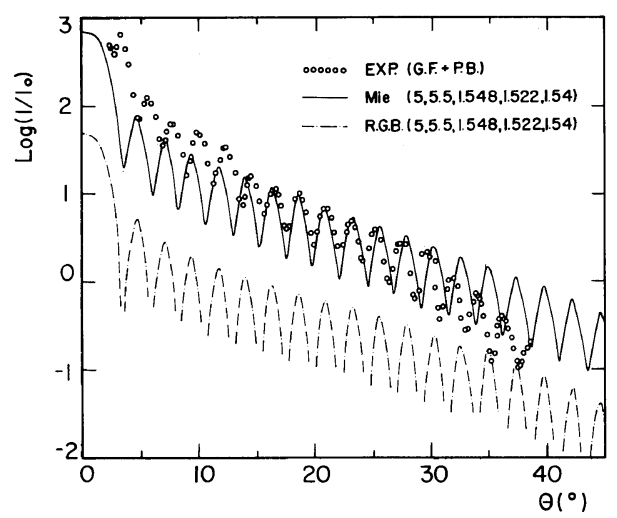

Figure 4. The experimental scattered intensity distribution for the polymer-coated glass fiber immersed in the silicone oil of refractive index $m_{3}=1.54$ (open circles) and the corresponding calculated Mie (solid line) and R.G.B. (dash-dot line) scattering intensity distributions. The numbers in parentheses refer to the same meaning as those in Figure 3. matrix in the FRR films subjected to the applied stress was also analyzed on the basis of the coaxial cylinder model.

Figure 5 shows typical phase contrast optical micrographs of the glass fiber $\left(R_{1}=5 \mu \mathrm{m}\right)$ embedded in the crosslinked polybutadiene films subjected to tensile elongation of (a) 0 , (b) 10 , (c) 40 , and (d) $60(\%)$ in a horizontal direction. By increasing the degree of stretching voids or density-lowered region

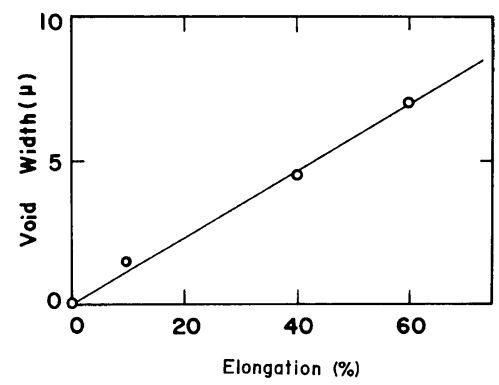

Figure 6. Change in the void width as a function of elongation percents of the FRR films.
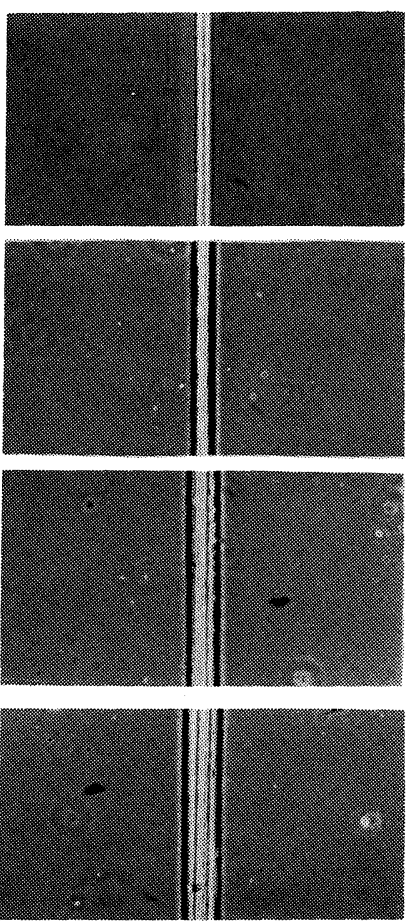

(c)

(b) 10

$\Delta R=1.5$

(a) $0(\%)$

$\Delta R=O(\mu)$

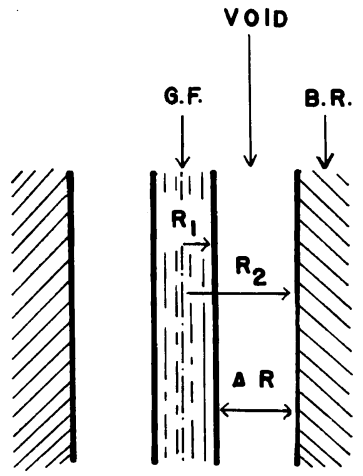

(d) 60

$\Delta R=12$

Figure 5. Typical phase-contrast micrographs of the FRR films streched by (a) 0 , (b) 10 , (c) 40 , and (d) $60 \%$ along the horizontal direction. 

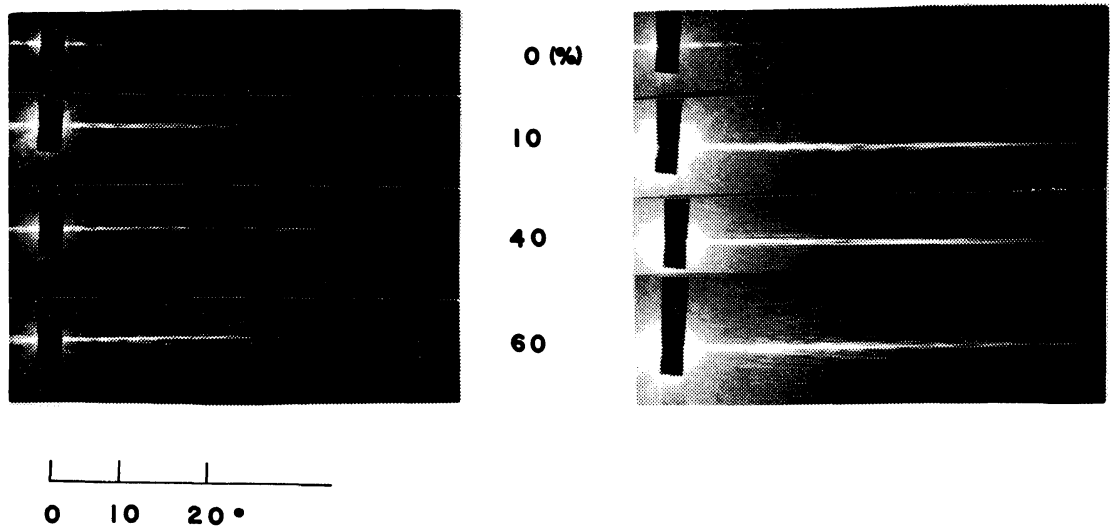

(a)

(b)

Figure 7. Typical photographic light scattering patterns from FRR films stretched by $0,10,40$, and $60 \%$ in the horizontal direction. The glass fiber is vertical. The patterns (a) are taken with an exposure time shorter than that for patterns (b).

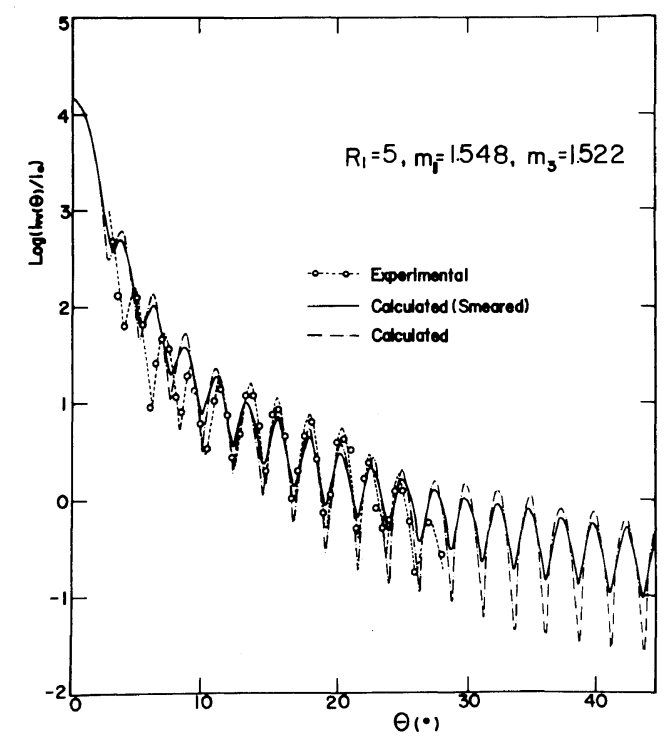

Figure 8. The photometrically measured intensity distribution of the unstretched FRR films (open circles) and the calculated smeared (solid line) and unsmeared (dashdot line) Mie scattering curves.

are generated at the interface between the fiber and matrix. The widths of the void phases increase almost linearly with the stretching percents as shown in Figure 6.

Figure 7 shows corresponding photographic $V_{\mathrm{v}}$ light scattering patterns obtained with short (a) and

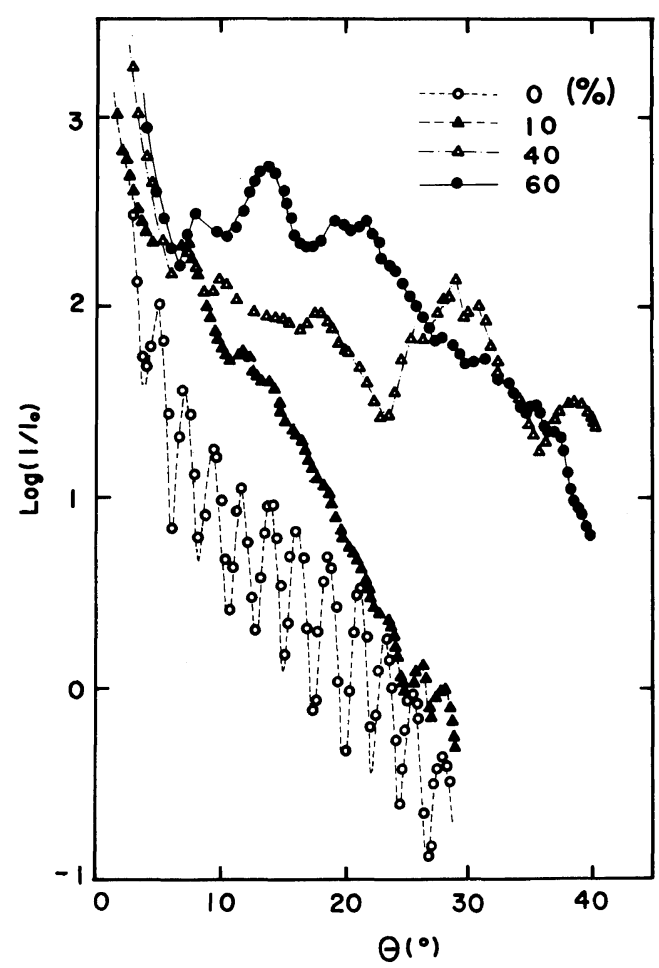

Figure 9. The photometrically measured intensity distributions of the FRR films stretched by $0,10,40$, and $60 \%$.

long (b) exposure times. The patterns change drastically even for small elongations and to exhibit a 
complex appearance with a series of fine striations parallel to the strong equatorial scattering (see Figure 7(b)). The striated patterns are similar to that for the coated fiber (Figure 2(b)). The scattered intensity increases with the elongation.

The photometrically measured equatorial intensity distributions of the patterns shown in Figure 7 are shown in Figure 8 for the unstretched FRR films (open circles) and in Figure 9 for the stretched films, (the intensity distribution for the unstretched film is also included for a reference). It is obvious from these results that the scattered intensity increases with stretching, especially at large angles, owing to the generation of a density-lowered or void region at the interface between the glass fiber and matrix.

\section{DISCUSSIONS}

In this section we will interprete the experimental scattering curves in terms of Mie or R.G.B. scattering from the coaxial cylinder model. It should be noted that all the calculated curves based on the Mie theory were smeared by finite angular resolution of the photometric apparatus ${ }^{2}$ in order to facilitate a direct comparison with the experimental curves.

\section{Polymer-Coated Glass Fiber}

In order to estimate the parameters $R_{1}, R_{2}, m_{1}$ and $m_{2}$ of the coated fiber, the scattering experiments were first conducted for the glass fiber alone to obtain its parameters $R_{1}$ and $m_{1}$, by the method described in detail in a previous paper, $\left(R_{1}\right.$ and $m_{1}$ were estimated to be $5.0 \mu \mathrm{m}$ and 1.548 , respectively). The parameters $R_{2}$ and $m_{2}$ of the polymer-coated sheath were evaluated by the method discussed in a previous paper. $^{3}$

The scattering intensity distributions were measured by immersing the coated fiber in a liquid whose refractive index was quite close to that of the fiber (as shown in the measured curve in Figure 4, for an example). Since the values of $m_{1}$ and $m_{3}\left(m_{1} \simeq m_{3}\right)$ are close to $m_{2}$, the scattering angles of the maxium intensity may be described by the R.G.B. theory.

The value of $\left(R_{1}+R_{2}\right)$ can be measured by using eq 10 . This value may also be estimated by measuring the angles of successive scattering maxima, e.g., $\theta_{m, i}$ and $\theta_{m, i+1}$ for $i$-th and $(i+1)$-th maxima on the basis of eq 12 , which is readily derived from eq 10 ,

$$
R_{1}+R_{2}=\frac{\lambda_{0}}{2 m\left[\sin \left(\theta_{m, i+1} / 2\right)-\sin \left(\theta_{m, i} / 2\right)\right]}
$$

The 10- and 11-th scattering maxima were detected at scattering angles $26.2^{\circ}$ and $28.5^{\circ}$ in the experimental curve in Figure 4, from which the value $\left(R_{1}+R_{2}\right)$ was estimated to be 10.5, (the value of $m$ was assumed to be 1.548). Since $R_{1}$ was determined as $5.0 \mu \mathrm{m}, R_{2}$ is thus $5.5 \mu \mathrm{m}$. This $R_{2}$ value obtained is in good agreement with that obtained by optical microscopy.

The refractive index of the sheath $m_{2}$ was also evaluated to be 1.522 by best-fitting the calculated Mie scattering curves obtained for various values of $m_{2}$ with the experimental curve, (other parameters $R_{1}, R_{2}, m_{1}$, and $m_{3}$ all being known at this stage). The calculated Mie scattering curve (solid line) for the set of parameters thus determined $\left(R_{1}=5.0\right.$, $R_{2}=5.5 \mu \mathrm{m}, m_{1}=1.548, m_{2}=1.522$ and $\left.m_{3}=1.54\right)$ is shown to be in good agreement with the measured curve. Figure 4 also indicates that the Mie scattering is almost identical to the R.G.B. scattering under the same conditions, since the R.G.B. criterion ${ }^{5}$ is satisfied by a good degree of accuracy. The intensity level of the R.G.B. scattering was arbitrarily shifted so as to avoid an overlap with the Mie curve.

In Figure 3, the measured scattering curve from the coated fiber in the air was compared with the calculated Mie and R.G.B. scattering for the set of parameters determined already. When the fiber was in the air, the difference in the refractive indices between the scattering material and the surrounding medium was so large that the R.G.B. criterion ${ }^{5}$ could no longer be satisfied, and thus gave rise to a large discrepancy between the R.G.B. scattering curve and the measured curve. The Mie scattering curve gives a better agreement with the measured curve rather than does the R.G.B. curve. Especially, the scattering angles of the intensity maxima agree fairly well with the experimental curve.

A discrepancy between the calculated and measured intensity distributions may arise from the fine striations occurring parallel to the main equatorial scattering as observed in the experimental patterns (see for an example Figure 2(b)). This discrepancy may be attributed to a low degree of inhomogeneity in the sheath thickness or the refractive index of the sheath. The inhomogeneity of the sheath may affect the scattering intensity distribution less by matching the refractive index of the surrounding medium with that of the sheath. Thus, better agreement between the calculated and measured scattering curves is expected to occur by immersing the fiber in silicone 
oil of $m_{3}=1.54$ as shown in Figure 4. Figure 3 also shows the Mie scattering from the glass fiber alone. The coated sheath is shown to decrease the scattering angles of the intensity maxima.

Effects of the immersion liquid on the scattering curve from the coated fiber are shown in the following. (i) the immersion liquid greatly reduces the scattering efficiency $Q_{V_{\mathrm{v}}}$, e.g., $Q_{V_{\mathrm{v}}}=2.33$ and 0.34 for $m_{3}=1.0$ and 1.54 , respectively, (ii) with the immersion liquid, the angular dissymmetry increases; and (iii) the scattering angles of the intensity maxima shift to smaller angles, and this may be described primarily as the effect of scattered light refraction at the interface between the coated fiber and the surrounding medium. ${ }^{2}$

\section{Light Scattering from a Debonded Phase of the FRR}

The experimental scattering curve from the unstretched FRR films was quantitatively compared with the calculated Mie scattering curve. The best fit of the calculated and experimental curves was again obtained for $m_{3}=1.522$ as in the case of the coated fiber. The values for $R_{1}=5.0 \mu \mathrm{m}$ and $m_{1}=1.548$ were predetermined according to the method discussed already. Figure 8 shows the results of the curve fitting and also the effect of smearing the calculated curve due to the finite angular resolution of the detecting system $^{2}$ (the smeared and unsmeared intensity distributions correspond to the solid and dash-dot lines, respectively).

A general trend observed for the change in the scattered intensity distributions upon stretching the FRR films is summarized as follows; (i) the sharp scattering maxima and minima observed in the unstretched films disappear with stretching, even for a small elongation, thus resulting in ill-defined broad maxima, and (ii) the scattered intensity increases with increasing stretching percents, especially at large scattering angles. The experimental results were analyzed on the basis of the simplified model based upon a coaxial cylinder model in which the sheath corresponds to the debonded interfacial phase.

Figure 10 shows the calculated Mie scattering curves for the coaxial cylinder having a sheath thickness corresponding to a void width of $0.1,2.0$, and $5.0 \mu \mathrm{m}$, i.e., $R_{2}=5.1,7.0$, and $10.0 \mu \mathrm{m}$. The scattering from the coaxial cylinders of $R_{2}=7.0$ and $10.0 \mu \mathrm{m}$ may roughly correspond to the 10 and $40-\%$ stretched films, respectively (Figures 5 and 6). The

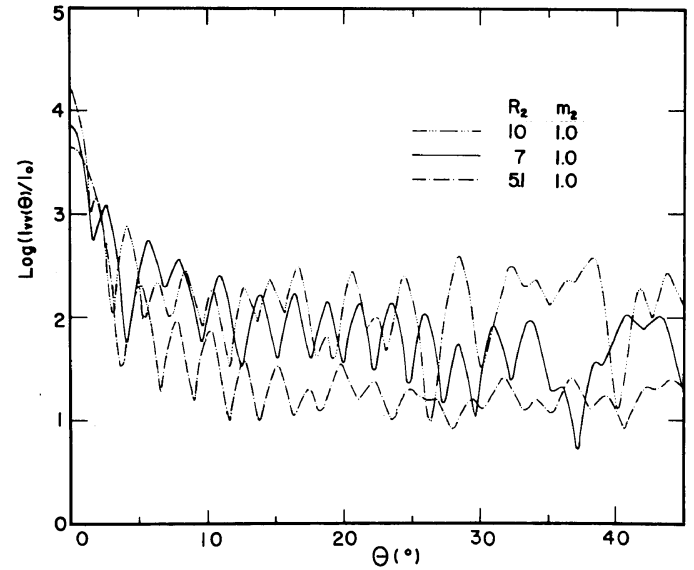

Figure 10. Calculated Mie scattering from the coaxial cylinder having $R_{1}=5.0 \mu \mathrm{m}, m_{1}=1.548, m_{2}=1.0$, and $m_{3}=1.522$ as a function of $R_{2}(\mu \mathrm{m})$.

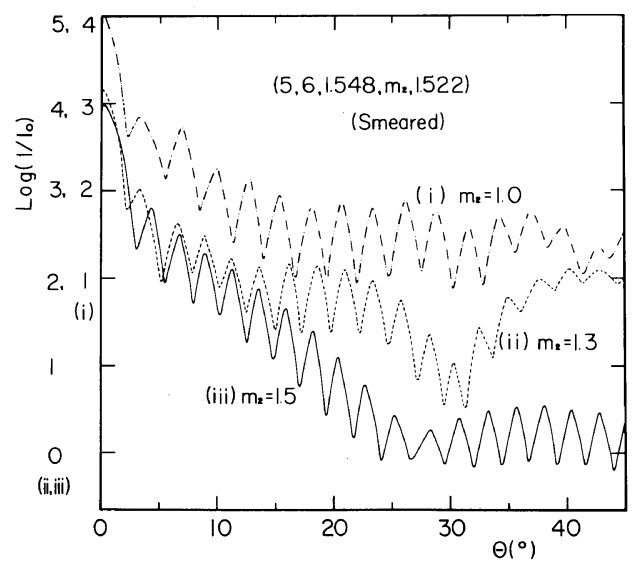

Figure 11. Calculated Mie scattering from the coaxial cylinder having $R_{1}=5.0 \mu \mathrm{m}, R_{2}=6.0 \mu \mathrm{m}, m_{1}=1.548$, and $m_{3}=1.522$ as a function of $m_{2}$.

refractive index of the sheath is assumed to be $m_{2}=1.0$, i.e., the sheath is a complete void (air phase). The parameters of the inner cylinder are those for the glass fiber, $R_{1}=5.0 \mu \mathrm{m}$, and $m_{1}=1.548$, and the refractive index of the medium $m_{3}$ is set to be 1.522 , i.e., the value estimated for the polybutadiene matrix.

Comparison of Figures 8 and 10 clearly indicates that even a thin air-sheath of $0.1 \mu \mathrm{m}$ drastically increases the scattered intensity, especially at large scattering angles and that the intensity further increases by increasing the sheath thickness. This is in good agreement with that found for the experim- 
ental curves (Figure 9). However, at a small strain, e.g., $10 \%$, the experimental scattering intensity does not increase as much as the calculated intensity for $R_{2}=5.1$ and $7.0 \mu \mathrm{m}$ increase, and its intensity distribution is a convex function (the second derivative of the function being negative) in contrast to the envelope of the calculated intensity distribution which is a concave function (the second derivative being positive).

The effect of varying the refractive index of the sheath $m_{2}$ on the intensity distributions was studied for a set of parameters $R_{1}=5.0, R_{2}=6.0 \mu \mathrm{m}$ (void width of $1.0 \mu \mathrm{m}$, roughly corresponding to the $10-\%$ stretched film) and $m_{3}=1.522$ in order to understand a possible origin of the discrepancy (Figure 11). With an increase in the refractive index of the sheath $m_{2}$, the scattered intensity increases less with the stretching and the envelope of the calculated intensity distribution changes from a concave function to a convex function in the angular range under consideration the tendencies of which are exactly in agreement with the experimental results. Thus, it is suggested that for small strains, e.g., $10 \%$, the refractive index of the sheath is greater than 1 owing to incomplete debonding of the polymer from the glass fiber. The sheath at small strains corresponds to the density-lowered phase at the interface between the fiber and the polymer matrix.

Another discrepancy between the calculated and measured intensity distributions stems from the fact that the experimental intensity distributions do not show the many sharp maxima and minima observed for the calculated distributions. This discrepancy was studied in terms of the size distribution of the outer radius $R_{2}$, the size distribution being assumed by the ZOLD function $P\left(R_{2}\right)$ in eq 7. An average relative $V_{\mathrm{v}}$ scattering $I / I_{0}$ was calculated by

$$
I / I_{0}=\int_{0}^{\infty} P\left(R_{2}\right)\left(I_{V_{v}}\left(\theta, R_{2}\right) / I_{0}\right) \mathrm{d} R_{2}
$$

where $I_{V_{v}}\left(\theta, R_{2}\right) / I_{0}$ is given by eq 1 .

Since we are considering the scattering from a single cylinder, the size distribution of $R_{2}$ should be related to the size distribution along the fiber. In general scattering from different parts of the fiber interfere with each other, so that the scattered intensity should actually be calculated by obtaining the scattered field from the coaxial cylinder having statistical fluctuations of the outer radius $R_{2}$. Since at

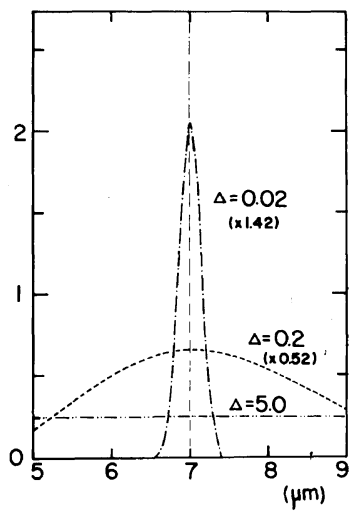

Figure 12. The truncated ZOLD functions used for the studies in Figure 13. $R_{\min }=5.0 \mu \mathrm{m}, R_{\max }=9.0 \mu \mathrm{m}$, and $R_{\mathrm{M}}=7.0 \mu \mathrm{m}$. The values in parentheses show the scale factor relative to the case of $\Delta=5.0$.

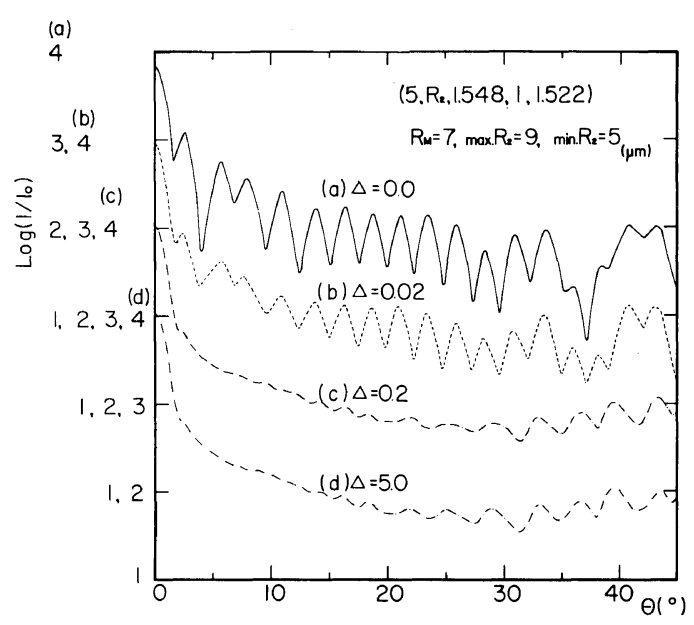

Figure 13. Effect of the size distribution in the outer radius $R_{2}$ on the calculated Mie scattered intensity distribution. $R_{1}=5.0 \mu \mathrm{m}, m_{1}=1.548, m_{2}=1.0$, and $m_{3}=1.522$ as a function of $\Delta$ in the distribution function. $R_{\mathrm{M}}=7.0 \mu \mathrm{m}$.

the present, calculation of the Mie scattering from such systems seems intractable, we assumed an additivity of the scattered intensity (thus neglecting the interference as in eq 13) from the portion of the fiber having different values of $R_{2}$, and this is of course a very crude approximation.

Figure 12 represents the normalized size distribution $P\left(R_{2}\right)$ that we will consider, the modal value $R_{\mathrm{M}}=7 \mu \mathrm{m}$, and the ZOLD function being truncated in such a way that the minimum and maximum 

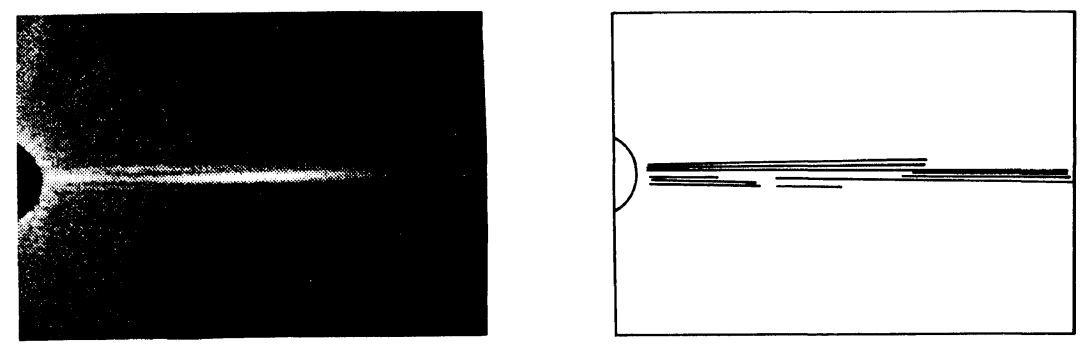

(a) $519 \mu n$
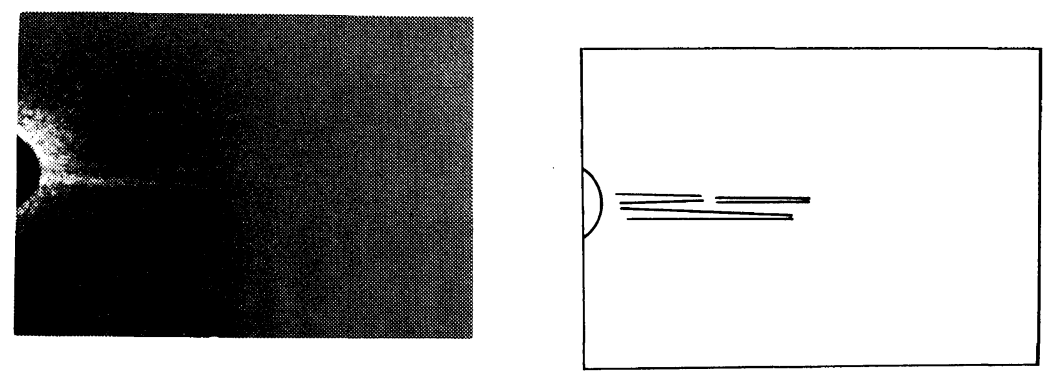

(b) $260 \mu \mathrm{m}$

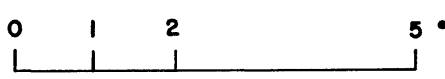

Figure 14. The photographic light scattering patterns and their schematic representations of the chopped glass fiber of lengths (a) 519 and (b) $260 \mu \mathrm{m}$.

values of $R_{2}$ are 5.0 and $9.0 \mu \mathrm{m}$, respectively. The numbers in parentheses show the scale factor for the case of $\Delta=5.0$.

Figure 13 shows the effect of the size distribution for the type shown in Figure 12 on the scattering from the coaxial cylinder having a set of the parameters $R_{1}=5.0 \mu \mathrm{m}, m_{1}=1.548, m_{2}=1.0, m_{3}=1.522$. The size distribution tends to smear effectively the intensity distribution corresponding to the monodisperse system with $\Delta=0$, which is in agreement with the experimental intensity distributions. By increasing the $\Delta$ value, i.e., the size distribution, the intensity maxima and minima tend to smear, while keeping intact the envelope of the curve except at small angles where the angular dissymmetry of the intensity distribution increases with increasing $\Delta$. The experimental scattering intensity decreases with the scattering angle by two to three decades for specimens stretched by 40 or 60 percents (Figure 9). This is observed for the calculated curves with the ZOLD type distribution with $R_{\mathrm{M}}=7.0 \mu \mathrm{m}$. However there still exist some discrepancies between the calculated and experimental curves. In the experimental curves, several broad irregular peaks can be seen, whereas the calculated curves with $\Delta=0.2$ or 5.0 give a smooth curve with the small and relatively periodic peaks at the large angle region. In order to improve the agreement between the calculated and experimental results, the statistical fluctuations of the sheath thickness and refractive index of the sheath should be treated in a more rigorous manner. In any case, it is obvious that the smearing of the scattering distribution of the stretched FRR films is attributed primarily to the distribution of the radii of the sheath corresponding to the void or density-lowered phase which could not be detected by a quick examination under optical microscopy.

The fine striations occurring parallel to the main equatorial scattering in the scattering patterns from the coated fibers (Figure 2) and the stretched FRR films (Figure 7) are considered to arise from inhomogeneities in the sheath thickness and the refractive index of the sheath along the fiber direction. This may be proved partly by observing the scattering patterns from the chopped glass fiber, since the chopped fiber represents a special form of the 
inhomogeneities of the refractive index of the fiber along its direction. The scattering patterns from the chopped fibers (Figure 14) clearly show fine striations parallel to the main equatorial scattering, just as those shown in Figures 2 and 7. The first maximum in the meridional direction occurs at scattering angles $\varphi=0.1^{\circ}$ (a) and $0.2^{\circ}$ (b), corresponding to fiber lengths of 519 and $260 \mu \mathrm{m}$, respectively, by using eq 6 , these results are in good agreement with the values estimated from optical microscopy.

The scattering profile is extremely sensitive to failure or debonding at the interface of a glass fiber from the polymer matrix when the fiber reinforced polymer solid is subjected to applied stress. Further refinement of the model and calculations of its Mie scattering may be useful for studying stress concentration and failure at the interface. Mie scattering is also useful in studying generation and propagation of the crazing and the cracking of rubber-toughened plastics.

\section{REFERENCES}

1. E. P. Plueddemann, Ed., "Composite Materials," Vol. 6, Academic Press, New York, N.Y., 1974.

2. Y. Uemura, M. Fujimura, T. Hashimoto, and $\mathrm{H}$. Kawai, Polym. J., 10, 341 (1978).

3. Y. Uemura, T. Hashimoto, and 'H. Kawai, Sen-I Gakkaishi, in press.

4. M. Kerker, "Scattering of Light and Other Electromagnetic Radiation," Academic Press, New York, N. Y., 1969.

5. H. C. Van de Hulst, "Light Scattering by Small Particles," John Wiley \& Sons, Inc., New York, N.Y., 1957. 